\title{
III.
}

Aus dem pharmakol. Institut von Prof. v. Schroeder in Heidelberg.

\section{Ueber Methylxanthin, ein Stoffwechselproduct des Theobromin und Coffeïn.}

Von

Dr. St. Bondzyński und Dr. R. Gottlieb.

Die Erkenntniss von der nahen chemischen Verwandtschaft der Körper der Xanthinreihe unter einander, besonders aber des Xanthins und Hypoxanthins zur Harnsäure hat für die physiologische Chemie durch die Beziehungen zur Frage der Harnsäurebildung ganz besonderes Interesse erlangt. Eine Reihe sowobl synthetisch-chemischer als physiologischer Untersuchungen wurden hierdurch angeregt, welche das Ziel verfolgten, einen directen Beweis für den Uebergang der Xanthinkörper in Harnsäure zu erbringen und so die Entstehung der Harnsäure im Organismus aufzuklären. Während die synthetische Chemie durch die Ueberführung von Dimethylharnsäure in Theophyllin von Emil Fischer und Ach ${ }^{1}$ ) diesem Ziele in neuester Zeit sehr nahe gekommen ist, haben die physiologischen Versuche bisher keineswegs einen sicheren Beweis für die Vermuthung erbracht, dass anch im Organismus der Säugethiere Harnsäure aus Xanthinkörpern entstehe. Zwar ist es Horbaczewski ${ }^{2}$ ) gelungen, ausserbalb des Organismus aus den Nucleïnen der Milzzellen Harnsäure zu erhalten, die Versuche im lebenden Thierkörper selbst haben aber keineswegs eindeutige Resultate ergeben (Stadthagen ${ }^{3}$ ), Gumlich ${ }^{4}$ ), Horbaczewski (l. c.), Richter ${ }^{5}$ ). Vielmehr scheint aus diesen Untersuchungen hervorzugehen, dass die Xanthinkörper dem Organismus

1) Berichte d. kgl. Akademie zu Berlin 1895.

2) Monatshefte für Chemie. Bd. X. S. 624.

3) Virchow's Archiv. Bd. CIX. S. 390.

4) Zeitschrift f. physiol. Chemie. Bd. XVIII. S. 50 s.

5) Zeitschr. f. klin. Med. Bd. XXVII. S. 290. 
einverleibt leicht weiter, vielleicht bis zu den nächsten Vorstufen des Harnstoffs zerstört werden.

Diese Gesichtspunkte haben uns die Anregung gegeben das Verhalten der Homologen des Xanthins, des Theobromins und Coffeïns im Organismus einer näheren Untersuchung zu unterziehen. Doch haben die Versuche zu einem völlig unerwarteten Resultate geführt.

Unsere Versuche knüpften an die im hiesigen Institute von Herrn Cand. med. E. Rost ${ }^{1}$ ) ausgefübrte Untersuchung über die Ausscheidung von Coffeïn und Theobromin aus dem Organismus an. Diese mit verlässlichen Methoden ausgeführten quantitativen Bestimmungen der beiden Xanthinderivate im Harn hatten ergeben, dass ein bedentend grösserer Antheil von Coffeïn und Theobromin der Zerstörung im Organismus entgeht und unverändert im Harn wieder erscheint, als man nach früheren Untersuchungen allgemein angenommen hatte. Auch in Bezug auf die vorhandene Literatur über die Ausscheidung der beiden Substanzen und die spärlichen, hauptsächlich negativen Angaben über die Form, in der sie nach ibrer Zerstörung den Organismus verlassen, dürfen wir auf die nachfolgende Abhandlung von E. Rost hinweisen.

Zur Darstellung des Umwandlungsproductes des Theobromins fübrten wir zwei Versuchsreihen an Kaninchen und eine am Hunde aus.

Versuch A. 3 Kaninchen erhielten mittelst Schlundsonde innerhalb 13 Tagen im Ganzen 27 g Theobromin anfangs in täglichen Gaben von $0,5 \mathrm{~g}$, später von $1 \mathrm{~g}$ in Emulsion mit etwas Gummi arabicum. Die während des Versuches gelieferte Harnmenge betrug $11,500 \mathrm{ccm}$.

Versuch B. In einer zweiten Fitterungsperiode wurden in gleicher Weise $52 \mathrm{~g}$ Theobromin in 19 Tagen an 4 Kaninchen verfuttert. Die Harnmenge betrug 16 Liter.

Versuch C. Endlich wurden einem grossen Hunde innerhalb 9 Tagen $24 \mathrm{~g}$ Theobromin mit Fleisch gegeben und die während der Versuchszeit gelassene Harnmenge von $6355 \mathrm{ccm}$ in Verarbeitung genommen.

Während die einmalige Gabe von $1 \mathrm{~g}$ Theobromin von Kaninchen mittlerer Grösse nicht ohne Weiteres vertragen wird, trat nach einigen Tagen bei Gaben von $0,5 \mathrm{~g}$ so weit Gewöhnung an das Theobromin ein, dass die tägliche Gabe nun auf $1 \mathrm{~g}$. gesteigert werden konnte, ohne dass eine acute Vergiftung eintrat. Oefters gingen die Thiere aber dennoch nach längerer Versuchsperiode unter Abnahme der Fresslust und chronischer Abmagerung zu Grunde.

Das verfütterte Präparat von Theobromin wurde vorher durch

1) Ueber die Ausscheidung des Coffeins und Theobromins aus dem Thierkörper. Von der medicinischen Facultät Heidelberg 1894 gekrönte Preisschrift. 
Stickstoffbestimmung anf seine Reinheit geprüft; die Bestimmung ergab 31,28 Proc. statt des berechneten 31,11 Proc. N.

Die in den drei Versuchsreihen erhaltenen Harnmengen wurden in der gleichen Weise aber getrennt verarbeitet. Die Untersuchungsmethode bestand in der Fällung des Harns mit Phosphorwolframsäure, Zersetzung des gut ausgewaschenen Niederschlages mit Barytbydrat und Ausfällung des Barytüberschusses mit Kohlensäure. Die barytfreie wässrige Lösung wurde dann auf dem Wasserbade zur Trockne abgedampft. Sie gab einen braunen, hygroskopischen, in Wasser leicht löslichen Rückstand. Derselbe wurde mit wenig Wasser gelöst und dabei von einer geringen Trübung (Baryumcarbonat) abfiltrirt. Eine kleine Probe der erhaltenen Lösung mit viel Ammoniak versetzt gab eine reichliche Fällung mit Silbernitrat, eine andere Probe einen reichlichen Niederschlag der Kupferoxydulverbindung. Da die beiden Reactionen dem Theobromin nicht zukommen, so lag unzweifelhaft ein vom Theobromin verschiedener Körper vor.

Auf die bekannte Fällbarkeit der Xanthinkörper als Kupferoxydulverbindungen hat neuerdings E. Drechsel ${ }^{1}$ ) aufmerksam gemacht und eine sehr sorgfältige Untersuchung der meisten Körper dieser Reihe durch $\mathrm{Balke}^{2}$ ) veranlasst. Da nach der Beobachtung von $P$. Balke Theobromin mit Kupferoxydulverbindungen nicht gefällt wird, so war damit die Möglichkeit der Trennung des Umwandlungsproductes von unverändertem Theobromin gegeben.

Zu der wässrigen noch warmen Lösung wurde eine Lösung von Kupfersulfat und Natriumbisulfit nach einem von M. Kruger ${ }^{3}$ ) in letzter Zeit ausgearbeiteten Verfahren so lange hinzugefiugt, bis kein Niederschlag mehr ausfiel und die dariber stehende Flüssigkeit deutlich grün gefärbt war. Der Niederschlag war flockig, anfangs grïnlich-weiss, später bräunlich. Nach 2-3 Stunden wurde derselbe filtrirt, ausgewaschen und mit einer nicht zu heissen Lösung von krystallisirtem Schwefelnatrium zerlegt. Das Filtrat vom Schwefelkupfer wurde mit Essigsäure angesäuert. Wenn die Lösung nicht zu verdünnt war, so fiel dadurch ein amorpher Niederschlag aus, der durch Kochen gelöst werden konnte. Die wässrige Lösung wurde weiter so lange erbitzt, bis aller Schwefelwasserstoff vertrieben war und endlich mit ammoniakalischer Lösung von Silbernitrat gefällt. Es fiel ein weisser, gelatinöser Niederschlag, welcher sich bald

1) Berichte der deutschen chem. Ges. Bd. XXV. S. 2454.

2) Journal f. prakt. Chemie. N. F. Bd. XLVII. S. 537.

3) Zeitschr. f. physiolog. Chemie. Bd. XVIII. S. 351. 
schwärzte ${ }^{1)}$ ohne aber dabei eine merkliche Zersetzung zu erleiden, und sich auch gat filtriren und auswaschen liess.

Der Silberniederschlag wird dann in Wasser suspendirt und in der Hitze durch Salzsäure zerlegt. Beim allmählichen Zusatz von verdünnter Salzsäure ballen sich die leichten Flocken zusammen und, wenn nach einigem Erhitzen die Reaction der Lösung deutlich sauer bleibt, besteht der am Boden des Gefässes sich ansammelnde, schwere Niederschlag nur aus Chlorsilber. Nach dem Abfiltriren wurde das Chlorsilber noch einige Male mit Wasser ausgekocht, die Filtrate vereinigt und eingedampft.

Bei stärkerer Concentration bedeckte sich die Oberfläche der Flitssigkeit mit Krusten und Knollen, die aus strablig zusammengesetzten Kugeln oder aus zu dichten Fächern vereinigten Nadeln bestanden. Das Gewicht der resultirenden, noch nicht gereinigten Substanz war im Versuch A etwa $3 \mathrm{~g}$, in Versuch B $12 \mathrm{~g}$, während aus dem Hundeharn nur $1,5 \mathrm{~g}$ des Rohpräparats erhalten wurden. Die Präparate A und B wurden auch weiterhin getrennt untersucht. In dem Präparate A wurden nach nochmaligem Umfällen mit Silbernitrat und einmaligem Umkrystallisiren die Analysen ausgeführt. Die aus Versuch B erhaltenen $12 \mathrm{~g}$ Substanz wurden zuerst der fractionirten Krystallisation unterworfen. Die erste Krystallfraction fiel aus etwa $1000 \mathrm{ccm}$ Lösung, während die letzte aus etwa $1 \mathrm{~g}$ Substanz bestand und durch allmähliches Einengen der Mutterlauge bis auf etwa $80 \mathrm{ccm}$ unter Trennung einiger Zwischenfractionen erhalten wurde. In der letzten Mutterlauge konnten nicht mehr als $0,1-0,2 \mathrm{~g}$ Substanz zurückgeblieben sein.

\section{Elementaranalysen des Umwandlungsproductes.}

Präparat aus Versuch A.

1. $0,2048 \mathrm{~g}$ Substanz ergaben $0,3286 \mathrm{~g} \mathrm{CO}_{2}$ und $0,0729 \mathrm{~g} \mathrm{H}_{2} \mathrm{O}$ entsprechend 43,71 Proc. $\mathrm{C}$ and 3,95 Proc. H.

2. $0,1554 \mathrm{~g}$ Substanz ergaben $43,8 \mathrm{~cm} \mathrm{~N}$ bei $11,8^{\circ} \mathrm{C}$. und $760 \mathrm{~mm}$ Barometerstand entsprechend 33,46 Proc. N.

3. 0,1751 g Substanz ergaben 0,4113 g Pt entsprechend 33,87 Proc. N (nach Will-Warrentrapp).

\section{Präparat aus Versuch B.}

Fraction a.

1. $0,2873 \mathrm{~g}$ Substanz ergaben $0,4612 \mathrm{~g} \mathrm{CO}_{2}$ und $0,1050 \mathrm{~g} \mathrm{H}_{2} \mathrm{O}$ entsprechend 43,66 Proc. C und 4,06 Proc. H.

1) Eher infolge von Abspaltung einer geringen Menge gebundenen Schweteis als durch eine Zersetzung des Silbersalzes. 
Ueber Methylxanthin, ein Stoffwechselproduct des Theobromin u. Coffeïn. 49

2. 0,3262 g Substanz ergaben $0,5212 \mathrm{~g} \mathrm{CO}_{2}$ und $0,1206 \mathrm{~g} \mathrm{H}_{2} \mathrm{O}$ entsprechend 43,57 Proc. C und 4,10 Proc. H.

3. $0,2261 \mathrm{~g}$ Substanz gaben $0,5254 \mathrm{~g}$ Pt entsprechend 33,50 Proc. $\mathbf{N}$ (Will-Warrentrapp).

4. $0,2153 \mathrm{~g}$ Substanz gaben $0,5024 \mathrm{~g}$ Pt entsprechend 33,64 Proc. $\mathbf{N}$ (Will-Warrentrapp).

Fraction b.

1. $0,2698 \mathrm{~g}$ Substanz ergaben $0,4277 \mathrm{~g} \mathrm{CO} 2$ und $0,0950 \mathrm{~g} \mathrm{H}_{2} \mathrm{O}$ entsprechend 43,23 Proc. $\mathrm{C}$ und 3,89 Proc. H.

\section{Fraction c.}

1. $0,3023 \mathrm{~g}$ Substanz ergaben $0,4806 \mathrm{~g} \mathrm{CO}_{2}$ und $0,1078 \mathrm{~g} \mathrm{H}_{2} \mathrm{O}$ entsprechend 43,21 Proc. C und 3,94 Proc. H.

2. $0,2106 \mathrm{~g}$ Substanz ergaben $0,4958 \mathrm{~g}$ Pt entsprechend 33,94 Proc. $\mathrm{N}$.

Präparat aus Versuch C.

1. $0,2419 \mathrm{~g}$ Substanz ergaben $0,3873 \mathrm{~g} \mathrm{CO}_{2}$ und $0,0900 \mathrm{~g} \mathrm{H}_{2} \mathrm{O}$ entsprechend 43,66 Proc. $\mathrm{C}$ und 4,13 Proc. $\mathrm{H}$.

2. 0,2022 g Substanz ergaben 0,4684 g Pt entsprechend 33,39 Proc. N.

In folgender Tabelle sind die Resultate der Elementaranalysen der einzelnen Präparate übersichtlich zusammengestellt:

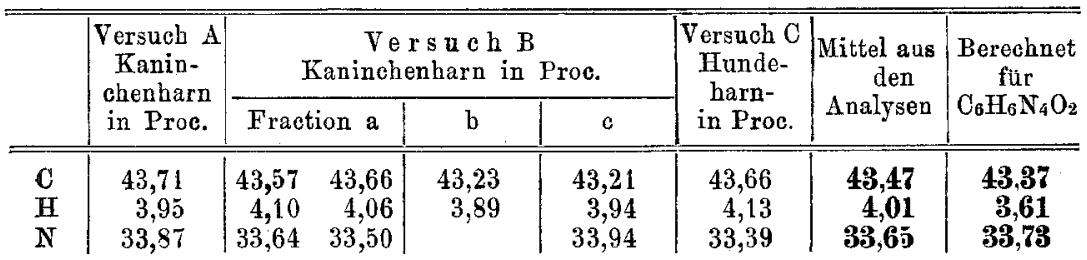

Aus den iubereinstimmenden Zahlen ergiebt sich die Formel $\mathrm{C}_{3} \mathrm{H}_{3} \mathrm{~N}_{2} \mathrm{O}$, welehe verdoppelt dem $\mathrm{Methylxanthin} \mathrm{C}_{6} \mathrm{H}_{6} \mathrm{~N}_{4} \mathrm{O}_{2}$ zukommt.

Eine Bestätigung der Annahme, dass die Formel mit 6 Kohlenstoffatomen die richtige sei, suchten wir zuerst durch die Moleculargewichtsbestimmung der Substanz zu erlangen. Bei der Schwerlös. lichkeit der Substanz in den ïblichen Lösungsmitteln bereitete dieselbe aber grosse Schwierigkeiten. Wir sind Herrn Prof. A uwers für seine freundlichen Rathschläge bei diesen Versuchen zu grossem Danke verpflichtet. Als das beste von den anwendbaren Lösungsmitteln erwies sich für das Umwandlungsproduct noch das Phenol. Doch musste erst durch Vorversuche ermittelt werden, ob die Xanthinkörper in diesem Lösungsmittel bei der Bestimmang der Gefrierpunktserniedrigung normale Werthe ergeben. Wir erhielten für Coffeïn, das in Phenol bei $45^{\circ} \mathrm{C}$. fast zerfliesslich ist, die Werthe 186 und 190 (statt des berechneten Moleculargewichts 194); für Theobromin, 
das gleichfalls von Phenol sehr leicht, wenn auch schwieriger als Coffeïn gelöst wird, ergab sich 186 und 180 (statt des Berechn. 180). Während diese Körper demnach normale Wertbe bei der Moleculargewichtsbestimmung nach der Methode der Gefrierpunktserniedrigung ergeben, gelangten wir bei dem viel schwerer löslichen Umwandlungsproducte - dasselbe löst sich nicht ganz 2 Proc. in Phenol bei $45^{\circ}$ C. - , nicht zum Ziele. Es ergaben die Bestimmungen vielmehr weit auseinanderliegende Werthe (268 und 98 statt des ftir Methylxanthin berechneten Werthes 166).

Dagegen ist es uns gelungen einige wohl charakterisirte $\mathrm{Me}$ tallverbindungen darzustellen und durch ihre Analyse die Annahme eines Methylxanthin zu erbärten. Wir haben ein Silbersalz, ein Baryumsalz und ein schön krystallisirendes Natronsalz der Substanz dargestellt.

Silbersalz. In Wasser gelöst giebt der neue Xanthinkörper mit Kupferoxydulsalzen eine flockige Fällung, mit ammoniakalischer Silberlösung einen im Ueberschuss von Ammoniak unlöslichen gelatinösen Niederschlag. Der Letztere gab mit Wasser gut ausgewaschen und anfangs über Schwefelsäure ${ }^{1}$ ), darauf bei $120-125^{\circ} \mathrm{C}$. bis zum constanten Gewicht getrocknet bei einer Silberbestimmung

$0,3166 \mathrm{~g}$ Substanz ergaben $0,1676 \mathrm{~g} \mathrm{Ag}$ entsprechend 52,9 Proc. Ag während eine dem Xanthinsilber analog zusammengesetzte Silberverbindung des Methylxanthins $\mathrm{C}_{6} \mathrm{H}_{6} \mathrm{~N}_{4} \mathrm{O}_{2} . \mathrm{Ag}_{2} \mathrm{O}$ 54,22 Proc. $\mathrm{Ag}$ verlangt. Diese Abweichung des gefundenen Werthes von dem berechneten geht zwar über die Feblergrenzen hinaus, erklärt sich aber durch die Beobachtung, die auch bei vielen anderen Xanthinkörpern gemacht wurde, dass ibre Silberverbindungen ein oder zwei Molectile Wasser sehr hartnäckig zurückzuhalten im Stande sind und dasselbe erst bei anhaltendem Trocknen bei Temperaturen von 130 oder $140^{\circ} \mathrm{C}$. verlieren. Eine geringe Schwärzung der Silberverbindung hat uns abgehalten sie höher zu erhitzen.

Natronsalz. In heisser Natronlange (im Verhältniss von zwei $\mathrm{Na}$ auf ein Molecul Methylxanthin) gelöst, gab der Körper eine Ausscheidung von centimeterlangen Krystallen, rhombische Tafeln und Säulen eines Natriumsalzes. Die Krystalle verwitterten beim Aufbewahren über Schwefelsäure. Lufttrocken gewogen zeigten sie nach dem Trocknen bei $100-105^{\circ} \mathrm{C}$. eine beträchtliche Gewichtsabnahme. Bis zur Gewichtsconstanz getrocknet ergaben bei der Natriumbestimmung

1) Die über Schwefelsäure staubtrocken gewordene Silberverbindung ergab bei der genannten Temperatur einen nicht unbeträchtlichen Gewichtsverlust. 
Ueber Methylxanthin, ein Stoffwechselproduct des Theobromin u. Coffein.

$0,2384 \mathrm{~g}$ Substanz ergaben $0,0879 \mathrm{~g} \mathrm{Na} \mathrm{SO}_{4}$ entsprechend 11,95 Proc. $\mathrm{Na}$ anstatt des für $\mathrm{C}_{6} \mathrm{H}_{5} \mathrm{NaN}_{4} \mathrm{O}_{2}$ Berechneten . . . . . 12,23 Proc. Na.

Die luftrockenen Krystalle weisen die Zusammensetzung $\mathrm{C}_{6} \mathrm{H}_{2} \mathrm{NaN}_{4} \mathrm{O}_{2}$ $+4 \mathrm{H}_{2} \mathrm{O}$ auf.

Baryumsalz. Weiters stellten wir ein Baryumsalz der Substanz dar. Die Lösung des neven Xanthinderivates in Natronlauge (die Natronlauge wurde frisch aus Natriummetall dargestellt) erstarrt bei Zusatz von Chlorbaryum zu einer Gallerte. Als dieselbe nach dem Abfiltriren und Abpressen in heissem Wasser gelöst wurde, gab die Lösung eine Ausscheidung des Baryumsalzes, die aus zu Kugeln und Rosetten vereinigten Krystallen bestand. Diese Krystalle fielen auch direct aus einer Lösung der Substanz in Barytwasser. Die Baryumbestimmung in einem einmal umkrystallisirten und bei 100 bis $105^{\circ} \mathrm{C}$. getrockneten Präparate ergab in

0,1883 g Substanz 0,0778 g BaCO 3 entsprechend 28,73 Proc. Ba anstatt des für $\left(\mathrm{C}_{6} \mathrm{H}_{5} \mathrm{~N}_{4} \mathrm{O}_{2}\right)_{2} \mathrm{Ba}$ Berechneten . . 29,34 Proc. Ba.

Es kann demnach keinem Zweifel unterliegen, dass die aus dem Theobrominharn dargestellte Substanz als Methylxanthin anzusprechen ist. Mit dieser Annahme stimmen auch die Löslichkeitsverhältnisse der Substanz gut iberein, da die Löslichkeit in Wasser, Alkohol und Chloroform dem Umwandlungsproducte unter den Xanthinderivaten die Stellung zwischen dem Theobromin einerseits und dem Xanthin andererseits anweist. Die Bestimmung der Löslichkeit ergab, dass sich lösen

$1 \mathrm{~g}$ der Substanz in $1592 \mathrm{ccm}$ Wasser bei $18^{\circ} \mathrm{C}$.

$\mathrm{ig}==\quad=109 \mathrm{ccm}=$ = Siedetemperatur

$1 \mathrm{~g}=\quad=\quad=7575 \mathrm{ccm}$ Alkohol absolutus bei $17^{0} \mathrm{C}$.

$1 \mathrm{~g}==\quad=2250 \mathrm{ccm}=\quad=\quad$ Siedetemperatur

Chloroform nimmt die reine Substanz nicht nachweisbar auf. In verunreinigtem Zustande dagegen löst sie sich ein wenig in Chloroform, aber auch dann bedeutend schwieriger als Theobromin. ${ }^{1}$ )

Endlich wurde die Annahme, dass wir ein Methylxanthin vor uns hatten, noch durch folgenden Versuch bestätigt, in dem es uns gelang durch Methylirung des Umwandlungsproductes Coffeïn zu erhalten.

$0,4 \mathrm{~g}$ ( 1 Mol.) bei $130^{\circ} \mathrm{C}$. getrockneten Silbersalzes wurden mit $0,8 \mathrm{ccm}$ Methylalkohol und der berechneten Menge (2 Mol.) Methyl-

1) So gelingt es bei richtiger Unterbrechung der Extraction mit Chloroform aus einem beide Substanzen enthaitenden Harnrückstande, das Theobromin von seinem Umwandlungsproducte einigermaassen zu trennen. 
jodid zusammengebracht und im zugeschmolzenen Rohre auf $100^{\circ} \mathrm{C}$. erhitzt. Schon nach einer Stunde war die Silberverbindung deutlich verändert. Nach 24 Stunden wurde der Inhalt der Röhre mit heissem Chloroform ausgezogen, der Chloroformauszug verdunstet. Er hinterliess einen Rückstand von einigen Centigramm, der nochmals in wenig kaltem Chloroform gelöst und daraus zurückgewonnen wurde; nun wurde mit wenigen Tropfen Wasser gelöst und die Lösung der freien Verdunstung überlassen. Bald bedeckte sich der Boden des Krystallisationsschälchens mit einem Beschlag von seideglänzenden Nadeln. Dieselben liessen sich obne Zersetzung leicht sublimiren. Die durch Sublimation gereinigten Krystalle schmolzen bei $226^{\circ} \mathrm{C}$, während ein Parallelversuch für die durch Sublimation aus dem käuflichen Coffeïn erhaltenen Krystalle an unserem Thermometer den Schmelzpunkt $229^{\circ}$ C. ergab. Die Krystalle waren in kaltem Wasser leicht löslich; die Lösung zeigte die We id el'sche Reaction in sehr intensiver Weise und gab keine Fällung mit Kupferoxydulsalzen. Dieser negative Ausfall der Kupferoxydulprobe kennzeichnet das Coffeïn wie das Theo. bromin unter allen anderen Xanthinkörpern (Balke l. c.) und unterscheidet es auch scharf von dem Methylxanthin.

Wenn auch die geringe Substanzmenge die Ausführung einer Elementaranalyse nicht gestattete, so kann es doch nach Krystallform und Löslichkeit, nach Schmelzpunktsbestimmung und Reactionen keinem Zweifel unterliegen, dass der aus dem Harn erhaltene Körper in Coffeïn übergeführt worden ist. Damit aber ist seine Zusammensetzung: als Methylxanthin erwiesen.

Theobromin wird demnach im thierischen Organismus in Methylxanthin nmgewandelt. Wie aus Kaninchenharn und Hundeharn konnten wir auch aus dem Harne des Menschen nach Einnahme von Theobromin einen Körper gewinnen, dessen Eigenschaften mit denen des Methylxanthins vollständig iubereinstimmten.

Es erübrigt uns noch eine kurze Beschreibung des neuen Körpers zu geben. In heissem Wasser gelöst fällt das Methylxanthin beim Erkalten der Lösung bald in Krusten, bald in Gestalt von kürzeren oder längeren mikroskopischen Säulen ans, bald krystallisirt es in halbeentimeterlangen Nadeln. Durchwegs aus solchen Nadeln bestand das Präparat aus Hundeharn. Zuweilen aber, besonders bei raschem Einengen der etwa noch gefärbten, verunreinigten Lösung fiel der Körper auch amorph aus. Ebenso wird er amorph, flockig durch Éssigsäure aus seiner Lösung in Alkalien ausgefällt; die Flocken wandeln sich aber bald in Krystalle um. Aus der Lösung 
Ueber Methylxanthin, ein Stoffwechselproduct des Theobromin u. Coffeïn. $\quad \mathbf{5 3}$ :

in Natronlauge wird Methylxanthin durch Ammoniaksalz wieder ausgefällt. Der Schmelzpunkt der Substanz liegt bis gegen $310^{\circ} \mathrm{C}$; das Schmelzen findet unter Zersetzung und Sublimation statt. Die Bestimmungen der Löslichkeit des Körpers in Wasser, Alkohol und Chloroform sind bereits oben angefiubrt.

Das Methylxanthin giebt die W eidel'sche Reaction sehr intensiv, nicht aber die sogenannte "Xanthinprobe". Von den Metallverbindungen der Substanz haben wir bereits gesprochen; mit Säuren salzartige Verbindungen einzugehen, scheint der Körper keine Neigung zu haben. Die Salzsåure verliert er wenigstens schon bei Wasserbadtemperatur vollständig.

Es entsteht weiter die Frage, ein wie grosser Antheil des Theobromins im Thierkörper in Methylxanthin übergeht. Dass es kein unbeträchtlicher Theil sei, liessen schon die Ausbeuten an Methylxanthin vermuthen, die wir in unseren Versuchen besonders in Versuch B erhielten. Noch besser geht dies aber aus Bestimmungen hervor, die wir zur Beantwortung dieser Frage ausführten. Die 48 stündige Harnmenge von Kaninchen wurde durch Abdrücken abgegrenzt und mehrmals der Untersuchung auf Xanthinkörper in der bei der Darstellung des Methylxanthins beschriebenen Weise unterworfen. Die nach der Zersetzung der Phosphorwolframsäureniederschläge erhaltene barytfreie Lösung wurde eingeengt und in einem Messkölbchen auf $200 \mathrm{ccm}$ gebracht. Je $100 \mathrm{ccm}$ der Lösung wurden nun einerseits mit ammoniakalischer Silberlösung, andererseits mit der erwähnten Kupferoxydulsalalösung gefällt; in beiden Niederschlägen wurde nach rollständigem Auswaschen der Stickstoff bestimmt. Es ergab sich durch diese mehrtägigen Bestimmungen, dass in Form von Xanthinkörpern in der 24 stündigen Harnmenge des Kaninchens $1 \mathrm{mg}$ Stickstoff ausgeschieden wird. Wurde nun der 48 stündige Harn nach Theobrominfütterung in gleicher Weise behandelt und die N-Bestimmung in den Niederschlägen ausgeführt, so entsprach die Stickstoffzunahme in dem Kupferoxydulniederschlag dem ausgeschiedenen Methylxanthin, die Stickstoffzunahme in dem nach Vertreiben des Ammoniaks ausgefallenen Silberniederschlag aber der Summe von Theobromin und Methylxanthin. Aus diesen Zahlen liess sich die Menge des ausgeschiedenen Theobromins and Methylxanthins berechnen. Ein solcher Versuch ergab nun, dass von dem verfütterten Theobromin $(1,5 \mathrm{~g})$ in den darauffolgenden 48 Stunden 19 Proc. The obromin unverändert im Harne wiedererschienen, während 24,6 Proc. zu Methylxanthin umgewandelt waren.

Methylxanthin ist auch wohl das einzige den ungespaltenen 
Xanthinkern noch enthaltende Umwandlungsproduct des Theobromins, denn die nach Verfütterung von $52 \mathrm{~g}$ Theobromin (Versuch B) erhaltenen $12 \mathrm{~g}$ Substanz bestanden bis auf einen geringen Rest von $0,1-0,2 \mathrm{~g}$, der nicht näher untersucht wurde, aus diesem Körper. Xanthin trat aber nicht auf.

Es bleibt vorläufig dahingestellt, in wie weit die eigenthümliche Abspaltung einer an ein Stickstoffatom gebundenen Methylgruppe eine im Thierorganismus verbreitete Erscheinung ist. Jedenfalls findet diese Abspaltung auch beim Coffeïn statt. Denn obgleich unsere Versuche über das Coffeïn noch nicht abgeschlossen sind, haben sie uns doch schon ergeben, dass nach Eingabe von Coffeîn im Harn der Versuchsthiere ein Körper erscheint, der in allen Eigenschaften vollständig mit dem Methylxanthin uibereinstimmt, dass also auch Coffer̈n im Organismus in Methylxanthin übergeführt wird. Doch erscheint von Coffeïn ein procentisch geringerer Antheil als Methylxanthin wieder als von Theobromin. Ob das Coffeïn bei dieser Umwandlung in Methylxanthin anch von der Vorstufe eines Dimethylxanthins begleitet ist, können wir noch nicht mit Bestimmtheit angeben; wir sind mit Versuchen beschäftigt, die darüber Aufschluss geben sollen.

Der Uebergang von Theobromin und Coffeìn in Methylxanthin bildet ein Gegenstïck zu der höchst interessanten von $\mathrm{Hofmeister}$ nachgewiesenen Umwandlung der Tellur- und Selensalze in Methyltellurid und Methylselenid ${ }^{1}$ ), besonders aber zu der von $\mathrm{His}^{2}$ ) beobachteten Umwandlung des Pyridins in Methylpyridin.

Methylirte Xanthinderivate sind nun anch als Bestandtheile des normalen Harns gefunden worden. Zuerst hat Thudichum ${ }^{3}$ ) ein solches unter dem Namen Urotheobromin beschrieben. Später wurde von G. Salomon ${ }^{4}$ ) ein von ihm als Paraxanthin benanntes Dimethylxanthin im Harne gefunden and näher gekennzeichnet, ein wohl mit Urotheobromin identischer Körper. Ferner wurde von S a lo m on ${ }^{5}$ ) aus Menschen- und Hundeharn ein Körper von der Zusammensetzung des Methylxanthins erhalten und Heteroxanthin genannt. Neuerdings wurde dasselbe auch von Balke (l. c.) nach Salomon's Methode aus dem Harne eines Leukämie-Patienten dargestellt und seine $\mathrm{Zu}$ sammensetzung als mit Methylxanthin übereinstimmend bestätigt. Dass

1) F. Hofmeister, Archiv f. exp. Path. u. Pharm. Bd, XXXIII. S. 198.

2) Ebenda. Bd. XXII. S. 253.

3) Compt. rend. CVI. p. 1803.

4) Archiv f. Anat. u. Phys. 1882. S. 426.

5) Berichte d. deutsch. chem. Ges. Bd. XVIII. S. 3400 . 
Ueber Methylxanthin, ein Stoffwechselproduct des Theobromin u. Coffeïn. 55

auch diese genannten Körper als secundäre Umwandlungsproduete im thierischen Organismus entstehen, dafür spricht der Umstand, dass sie nur in äusserst geringen Mengen aus dem Harn erhalten wurden, und dass man sie niemals als Bestandtheile der Zellkerne neben den bekannten Xanthinkörpern gefunden hat. Es fehlt uns an genügenden Beweisen, um die Identität des Heteroxanthins mit unserem Methylxanthin zu behaupten, doch können wir die Vermuthung nicht zurückweisen, dass auch das Heteroxanthin durch Abspaltung der Methylgruppe aus einem höher methylirten Xanthinderivat hervorgeht, das mit der Pflanzennahrung in den Organismus gelangt. Dadurch würde sich vielleicht der Befund des Heteroxanthin im Harn erklären. ${ }^{1)}$

Nachdem wir das Methylxanthin als ein Umwandlungsproduct von Coffeïn und Theobromin im Organismus genügend chemisch charakterisirt za haben glauben, wird es nunmehr von Interesse sein die Beziehungen dieses Umwandlungsproducts zur diuretischen Wirkung der Muskelsubstanzen und sein sonstiges physiologiscbes Verhalten festzustellen.

Heidelberg;, Mai 1895.

1) G. Salom on, Zeitschr. f. physiol. Chemie. Bd. XI. Dem Versuch am Hunde entnehmen wir, dass die Nahrung "aus Fleisch und Gemüse bestand." 